\title{
OBSERVATION OF QUADRUPOLE WAKE-FIELD EFFECTS IN THE KEKB LINAC
}

\author{
Y. Ogawa and T. Suwada, KEK, Tsukuba, Japan
}

\begin{abstract}
The characteristics of quadrupole wake-field effects were investigated using a single-bunch, high-intensity primary electron beam for positron production at the KEKB linac. $\mathrm{X}-\mathrm{Y}$ coupling phenomena in transverse motion, which are inherent in the instability due to quadrupole wake-field effects in linacs, were examined; their dependence on the initial beam kicks was measured as a quadratic nature. The results are in good agreement with a simple calculation based on a two-particle model.
\end{abstract}

\section{INTRODUCTION}

An investigation of wake-field effects in linacs will not only be definitely necessary for designing future linear colliders, but also even more essential for stably operating the existing high-intensity electron linacs, such as the KEKB linac[1], where the charge of single-bunched, primary electron beams for positron production already amounts to 10 nano Coulomb. In these situations, even quadrupole wake-field effects could emerge as observable phenomena [2] as well as the usual dipole wake-field effects, which might give a severe constraint on establishing the stable operation of high-intensity linacs. We have so far examined the characteristics of quadrupole wake-field effects using single-bunched, high-intensity electron beams at the KEKB linac. In a previous report [2], we discussed the $x-y$ coupling phenomena in betatron motions inherent in quadrupole wake-field effects, while addressing the first observation of the quadrupole wakefield instability in electron linacs. In this paper the results on more elaborate beam tests are reported, showing the quadratic nature of quadrupole wake-field effects on given transverse kicks of the beam orbit.

\section{WAKE-FIELD EFFECTS IN A TWO- PARTICLE MODEL}

A single-bunched electron beam in transverse space, which is accelerating under a constant gradient $(g)$ with an initial energy of $\gamma_{0}$ in the presence of an external smooth focusing system, is well described by the following equations of motion for a two-particle model [3]:

$$
\frac{d}{d s}\left(\gamma \frac{d X_{i}}{d s}\right)+\gamma k^{2} X_{i}=F_{i},
$$

where $X_{i}$ and $F_{i}$ are the transverse vectors of the displacements and forces for either a leading or trailing particle $(i=1,2), \gamma=E / m c^{2}=\gamma_{0}+g s, E$ is energy, $m$ the electron mass, $c$ the speed of light, $k$ the betatron wave number, and $s$ the distance down the linac. The external forces $\left(F_{i}\right)$ caused by transverse wake fields are explicitly written in the case of a round beam:

$$
\begin{aligned}
F_{1} & =0, \\
F_{2} & =-\frac{N r_{0}}{2}\left(W_{d} D_{1}+2 W_{q} Q_{1} X_{2}\right), \\
D_{1} & =\left[\begin{array}{l}
x_{1} \\
y_{1}
\end{array}\right]=X_{1}, \\
Q_{1} & =\left[\begin{array}{cc}
Q_{n} & Q_{s} \\
Q_{s} & -Q_{n}
\end{array}\right]=\left[\begin{array}{cc}
x_{1}^{2}-y_{1}^{2} & 2 x_{1} y_{1} \\
2 x_{1} y_{1} & y_{1}^{2}-x_{1}^{2}
\end{array}\right],
\end{aligned}
$$

where $W_{d}$ and $W_{q}$ are the dipole and quadrupole wake fields at the trailing particle $(i=2)$ generated by the leading particle $(i=1)$ with dipole and quarupole moments of $D_{1}$ and $Q_{1}, N$ the total number of particles in a bunch, and $r_{0}$ is the classical electron radius $r_{0}=2.818$ $\left.10^{-15} \mathrm{~m}\right)$.

The distinct features concerning the quadrupole wakefield effects in the force term $F_{2}$ are:

- $\quad x-y$ coupling phenomena directly originated in the skewed component of the quadrupole matrix, $Q_{s}$;

- parametric excitation due to the normal component of the quadrupole matrix, $Q_{n}$;

- quadratic dependence of the above-mentioned effects on the initial kicks.

The above classification into $\mathrm{x}-\mathrm{y}$ coupling and parametric excitation phenomena is just conceptual, and both effects are mutually dependent so that, for instance, the parametric excitation could also induce observable $x-y$ coupling effects in an actual situation. Therefore, the usual measurements on quadrupole wake-field effects are carried out while giving a large initial kick to one transverse direction and a small initial kick to the other transverse direction (possibly some orbit fluctuations), and observing the betatron oscillation in both directions [2]. This condition corresponds to the case $Q_{n}>>\mathrm{Q}_{\mathrm{s}}$, where both $x-y$ coupling effects through parametric excitation phenomena and genuine parametric-excitation effects might be observable at the same time.

The $x-y$ coupling effects in this context were already observed for single-bunched, high-intensity electron beams at the KEKB linac [2], while their quadratic dependence on the initial kicks as well as an observation of the genuine parametric-excitation effects has not been experimentally verified yet, which is the main issue of this paper. 


\section{EXPERIMENTAL}

An observation of the quadrupole wake-field effects was carried out in the first two sectors of the KEKB linac, where the regular beam optics with a constant betatron wavelength of about $46 \mathrm{~m}$ is realized so that a smooth focusing approximation could be appropriate. Figure 1 shows a schematic view of the corresponding section. A single-bunched electron beam with a charge of several nano Coulombs and a bunch length of $3 \mathrm{~mm}$ (FWHM) coming from the bunching section goes into the regular accelerator section through a matching section. The initial kicks are given at an energy of $230 \mathrm{MeV}$ and the transverse motion of the beam is observed downstream. The typical horizontal betatron oscillation is shown in Figure $2 \mathrm{a}$, when a horizontal initial kick of $0.3 \mathrm{mrad}$ is set; the vertical betatron oscillation induced by $\mathrm{x}-\mathrm{y}$ coupling effects due to quadrupole wake-fields can be seen in Fig. 2b [2]. The solid curve indicates simulation results using the parameters listed in Table I, where the values, except for the wake-field strengths which are used as fitted parameters, are observed with independent methods. The agreement between the observations and the simulations is quite satisfactory within the experimental errors.

Table 1: Linac parameters.

\begin{tabular}{|l|c|c|}
\hline \multicolumn{1}{|c|}{ Parameter } & Symbol & Value \\
\hline Number of Particles & $N$ & $510^{10}(8 \mathrm{nC})$ \\
\hline Bunch Length & $\sigma_{\mathrm{z}}$ & $1.5 \mathrm{~mm}$ \\
\hline Betatron Wave Number & $k$ & $2 \pi / 461 / \mathrm{m}$ \\
\hline Initial Energy & $\gamma_{0}$ & $230 / 0.511$ \\
\hline Energy Gain & $g$ & $20 / 0.5111 / \mathrm{m}$ \\
\hline Dipole Wake & $W_{1}\left(2 \sigma_{\mathrm{z}}\right)$ & $4.510^{5} 1 / \mathrm{m}^{3}$ \\
\hline Quadrupole Wake & $W_{2}\left(2 \sigma_{\mathrm{z}}\right)$ & $5.010^{9} 1 / \mathrm{m}^{5}$ \\
\hline
\end{tabular}

In order to investigate the quadratic dependence of the $x-y$ coupling effects on the initial kick, we measured the variation of the horizontal orbit at two fixed points in the linac by changing the initial vertical kick. The result is shown in Fig. 3, clearly indicating the quadratic behaviour as a function of the initial kick. The linear term in the fitted second-order polynomial manifests the dipole wake-field effects, while the quadratic term proves the quadrupole wake-field effects.

We also examined the quadratic dependence of genuine parametric excitation while giving the vertical initial kick, and observing the variation in the same vertical orbit at a fixed point down the linac. Figure 4 shows the result, indicating again the quadratic dependence on the initial kick, even though it is rather weak compared with the $x-y$ coupling case.
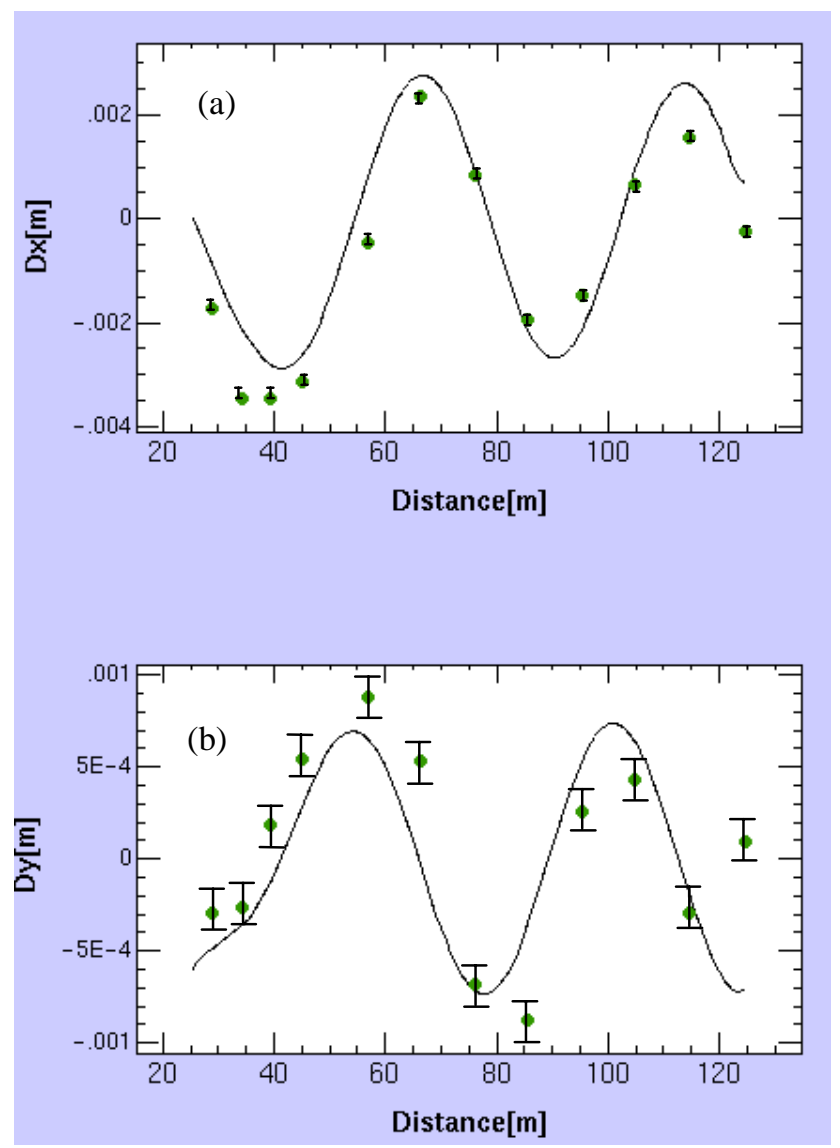

Fig. 2: X-Y coupling effects through the parametric excitation observed in the vertical orbit when a horizontal kick of about $0.3 \mathrm{mrad}$ was set to an $8-\mathrm{nC}$ beam at the initial point with an energy of $230 \mathrm{MeV}$ $(s=25.4 \mathrm{~m})$. The solid line indicates the simulation results using the parameters listed in Table I.

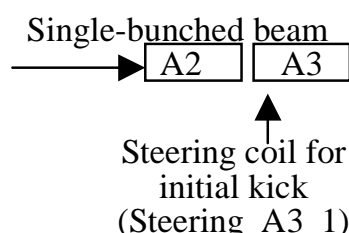

(Steering_A3_1)

Accelerator units: Sectors A - B

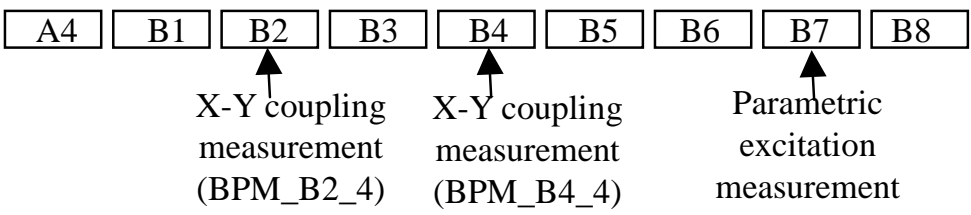

Fig. 3a Fig. 3b
(BPM_B7_4)

Fig. 4

Betatron oscillation measurement (100 m) Fig. 2

Fig. 1: Schematic view of the experimental section at the KEKB linac. 

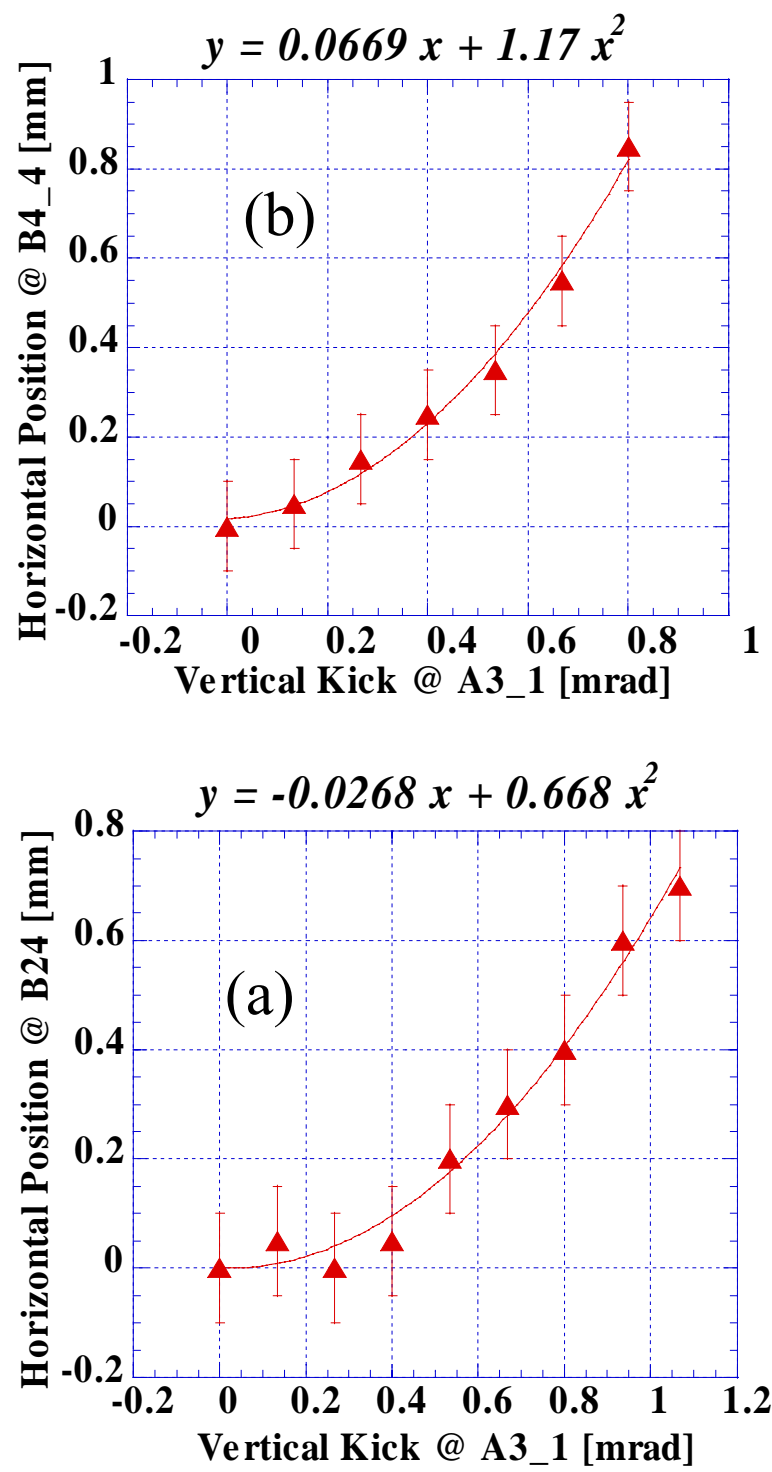

Fig. 3: Two examples of the quadratic dependence of the $x-y$ coupling effects through parametric excitation on the initial kick. The horizontal variations of the beam positions at the middle of the sector B2 (a) and the sector B4 (b) were observed by changing the initial vertical kick at the same position as in Fig.2.

\section{DISCUSSIONS AND CONCLUSIONS}

In series of experiments concerning the quadrupole wake-field effects performed at the KEKB linac, we could verify that the observed phenomena are really inherent in the quadrupole wake-field effects. A more detailed analysis as well as experiments, however, should be followed up in order to clarify the important nature of the quadarupole wake-field effects in linacs.

The following items are under consideration for the present:

- A precise beam-tracking simulation;

- An elaborate evaluation of the wake-field strength in each accelerator section;

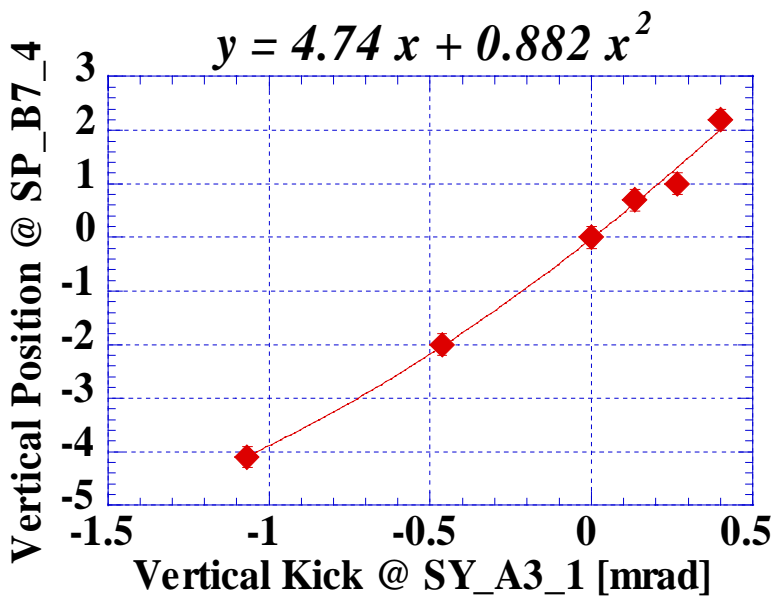

Fig. 4: The quadratic dependence of the genuine parametric-excitation effects on the initial kick. The horizontal variation of the beam position at the middle of the sector B7 was observed by changing the initial vertical kick at the same position as in Fig.2.

- Fine measurements using two-bunched beams with variable bunch separation.

In two-bunched beams, which are being extensively studied and tested at the KEKB linac for increasing the total charge of a primary electron beam in order to double the positron intensity [4], multi-bunch wake-filed effects might emerge as more important phenomena. Changing the bunch separation, we could see the variation in the wake-field strength generated by the leading bunch at the trailing bunch. In this way, we might expect an experimental distinction between the dipole and quadrupole wake-field effects, utilizing the different frequency spectra of the wake fields. Inhibiting either the leading bunch or trailing bunch acceleration with a proper bunch selection system, we also could obtain more fruitful information concerning the wake-field instability in linacs.

The authors wish to thank $\mathrm{K}$. Oide for valuable discussions. They have also greatly benefited from important suggestions concerning the experiment by $\mathrm{Y}$. Funakoshi.

\section{REFERENCES}

[1] T. Suwada et al., "Present Status and Beam Stability Issues of the KEKB Injector Linac", in these proceedings.

[2] Y. Ogawa, "Quadrupole Wake-Field Effects in the KEKB Linac" EPAC'00, Vienna, June 2000.

[3] P. B. Wilson, "High Energy Electron Linacs: Applications to Storage Ring RF Systems and Linear Colliders", in Physics of High Energy Particle Accelerators, AIP Conference Proceedings No.87, p.450, AIP, New York, 1982.

[4] S. Ohsawa et al., "Increase of Positrons by HighIntensity Two Bunches at the KEKB Linac", in these proceedings. 